\title{
Reconstructions of GaN and InGaN Surfaces
}

\author{
R. M. Feenstra, Huajie Chen, and V. Ramachandran \\ Department of Physics, Carnegie Mellon University, Pittsburgh, Pennsylvania \\ 15213 USA \\ A. R. Smith \\ Department of Physics and Astronomy, Ohio University, Athens, Ohio 45701 USA \\ D. W. Greve \\ Department of Electrical and Computer Engineering, Carnegie Mellon University, \\ Pittsburgh, Pennsylvania 15213 USA
}

\begin{abstract}
The reconstruction and growth kinetics of gallium nitride (0001) and $(000 \overline{1})$ surfaces are studied using scanning tunneling microscopy, reflection high-energy electron diffraction, and low-energy electron diffraction. Results for bare GaN surfaces are summarized, with particular attention paid to the "pseudo- $1 \times 1$ " reconstruction of the (0001) face. Changes in the surface structure and kinetic processes due to indium co-deposition during growth are discussed.
\end{abstract}

\section{Introduction}

Despite remarkable progress in device development using $\mathrm{GaN}$ and related alloys over the past number of years, relatively few studies have been performed on the fundamental surface properties of the material. Studies of the atomic-scale structure of the surface are important from the point of view of understanding growth of the material. Also, observation of surface reconstructions can be used to determine the polarity of the material [1]. As discussed below, the surface geometry affects kinetic processes during growth, presumably leading to variations in quality of grown films which in turn may affect device operation. Thus, it is important to determine the fundamental surface arrangements which occur on the surface.

Very early work on GaN surfaces using scanning tunneling microscopy (STM) was reported by Packard, Dow and co-workers [2]. More comprehensive work combining STM and firstprinciples theoretical computations was used by our group to identify the surface reconstructions for both the Ga-polar (0001) and the N-polar (0001) surfaces, prepared by molecular beam epitaxy (MBE) [3-5]. Detailed work by Held et al. using thermal desorption spectroscopy has provided valuable information on the gallium coverage of GaN during growth [6]. Photoemission spectroscopy has revealed the presence of well defined dangling bond states on the surface, although the geometry (and polarity) of the surfaces studied there was not clear $[7,8]$.

Work in our group over the past several years has identified the two families of surface reconstructions associated with the two c-plane surfaces of hexagonal GaN: the (0001) surface and the $(000 \overline{1})$ surface [1,3-5]. Reconstructions on both types of surfaces are quite remarkable in that they consist of metallic layers of gallium, bonded onto the GaN. This is an unusual situation for two reasons: first, most semiconductor surfaces prefer to be non-metallic, since the opening up of a surface gap provides a mechanism for energy lowering of the system. Second, the separation of 
the Ga atoms in bulk Ga is typically $2.7-2.8 \AA$, considerably smaller than the lattice constant of GaN, $3.19 \AA$. Nevertheless, at least for the $(000 \overline{1})$ surface, the Ga atoms on the surface form a $1 \times 1$ structure with a Ga-Ga separation of $3.19 \AA$, as pictured in Fig. 1(a). Charge transfer from Ga to N atoms stabilizes this structure (the Ga atoms in the adlayer are slightly positively charged so that there is a Coulombic repulsion between them). Additional reconstructions, with $3 \times 3,6 \times 6$, and $\mathrm{c}(6 \times 12)$ symmetry, are produced by depositing sub-monolayer quantities of Ga onto this $1 \times 1$ surface, as pictured in Fig. 1(b). The additional Ga adatoms are weakly bound on top of the $1 \times 1 \mathrm{Ga}$ adlayer, and consequently these higher order reconstructions are stable only below $\approx 300^{\circ} \mathrm{C}$ above which they undergo reversible order-disorder phase transitions [9].

For the (0001) surface, in the N-rich limit, we find that the surface is terminated with an arrangement of adatoms with each adatom forming three bonds to underlying Ga atoms, as pictured in Fig. 1(c). Probably both $\mathrm{N}$ and Ga species are involved as adatoms; several different structures have been observed including $2 \times 2,5 \times 5$, and $6 \times 4$, with the former two believed to consist of simple adatom arrangements and the latter one apparently being a more complicated reconstruction [5]. (For the $2 \times 2$, considerable confusion exists in the literature since there are many reports of this reconstruction but the majority of these involve, we believe, arsenic contamination of the surface $[10,11])$. In the Ga-rich limit, we find that there are two monolayers of Ga on top of the Ga-terminated bilayer [4], with these Ga layers assuming something close to their bulk spacing so that they form an incommensurate structure on the surface, as pictured in Fig. 1(d). The Ga atoms in the adlayers prefer their bulk spacing in this case because the bonding to the Ga-layer in the top GaN bilayer is relatively weak, in contrast to the $(000 \overline{1})$ surface where much stronger bonds are formed between the Ga adlayer and the underlying N-layer. We denote this reconstruction as " $1 \times 1$ " (in quotation marks) or pseudo- $1 \times 1$.

In this paper we discuss surface reconstructions, and their effects on the kinetics of film growth, for bare and indium-covered $\mathrm{GaN}$ surfaces. We first discuss some previously unpublished results for the pseudo- $1 \times 1$ surface of the Ga-face which lend support to the above mentioned model of its structure. We then examine the kinetics of growth by observing surface morphologies in the Ga-rich and N-rich regimes during growth. Results for the bare surface are found to differ compared to those obtained with indium overlayers; data for that case is presented, and the effects of the overlayer on the growth kinetics are discussed.

\section{Experimental}

Details of the growth are similar to those previously given [3-5], and will be briefly summarized here. GaN films are grown by $\mathrm{MBE}$ at a temperature of typically $700^{\circ} \mathrm{C}$ using a gallium effusion cell and rf-plasma nitrogen source. N-polar films are grown on sapphire, and Ga-polar films are grown on $\mathrm{Si}$-polar $6 \mathrm{H}-\mathrm{SiC}(0001)$ substrates. The substrates are prepared $e x$-situ by hydrogen etching [12]. InGaN films are grown at temperatures around $650^{\circ} \mathrm{C}$, using an effusion cell for the indium source. Typical growth rates for $\mathrm{GaN}$ and $\mathrm{InGaN}$ are $200 \mathrm{~nm} / \mathrm{h}$. In and Ga flux rates are calibrated with an in situ crystal thickness monitor, and the substrate temperature is measured by an optical pyrometer with emissivity set to 0.7 .

\section{3 " $1 \times 1$ " structure of the (0001) surface}

The structural model for the " $1 \times 1$ " structure of the (0001) GaN surface described above has been developed mainly on the basis of diffraction observations, using reflection high-energy electron 
diffraction (RHEED) and low-energy electron diffraction (LEED) [4]. STM imaging of the " $1 \times 1$ " surface was performed, but it did not yield the expected incommensurate pattern (i.e. series of fringes) expected for this surface. Furthermore, the atomic spacing which was observed corresponded accurately to the true $1 \times 1$ spacing $(3.19 \AA)$ as opposed to a value $1 / 6$ or $1 / 12$ smaller than this (as implied by the diffraction results). An explanation for this apparent discrepancy was proposed, namely, that the Ga atoms in the double layers were undergoing rapid motion so that the STM sensed the underlying corrugation of the GaN lattice. Supporting evidence for such atomic motion came from the diffraction results, which indicated a dynamic, fluidlike behavior of the overlayer at room temperature and above. This result is not so surprising given the low melting point of bulk $\mathrm{Ga}\left(29.8^{\circ} \mathrm{C}\right)$.

Although the observations described above do provide, we believe, a substantial basis for the " $1 \times 1$ " structural model, it is nevertheless desirable to have direct, real-space confirmation of this structure. We present here some data which may provide that type of confirmation. Shown in Fig. 2 are STM images acquired from the $(000 \overline{1})$ surface of GaN. On this surface we do not of course expect to find the " $1 \times 1$ " reconstruction, unless an inversion domain exists in the crystal and extends up to the surface. Transmission electron diffraction studies of our N-polar material grown on sapphire indicate that we do indeed have a moderate density of such inversion domains. As seen in Fig. 2(a), a large pit or hole is present in the film. We associate this pit with an open-core dislocation of the type previously seen in GaN films grown on sapphire [13]. The Burgers vector of those dislocations was $\mathbf{c}=0.52$ (0001) $\mathrm{nm}$. In the image of Fig. 2(a), two steps can be seen extending out of the pit, each with step height of $0.26 \mathrm{~nm}$, thus giving an (0001) component of the Burgers vector in agreement with the prior observations. (We note that, inside the pit of Fig. 2(a), convolution between probe-tip and sample topography take place which probably accounts for the apparent narrowing of the hole as one moves inside it).

Surrounding the pit seen in Fig. 2(a) are regions of $6 \times 6$ and $c(6 \times 12)$ reconstructed surface; these are the reconstructions commonly observed on the $(000 \overline{1})$ surface grown under relatively Ga-rich conditions. However, several small regions around the pit have distinctly different structure - the largest of these is indicated as $\approx 12 \times 12$ in Fig. 2(a). Expanded views of this region are shown in Figs. 2(b) and (c). This novel reconstruction has an appearance unlike any seen previously on the $(000 \overline{1})$ surface. It consists of a modulated structure with three-fold symmetry and nearly $12 \times$ spacing, and with substructure within each unit cell of nearly $1 \times$ spacing. Thus, this novel structure has precisely the characteristics which we associate with the " $1 \times 1$ " structure of the (0001) surface.

To associate the observed novel reconstruction with the " $1 \times 1$ " structure would imply the existence of an inversion domain immediately below that area of the surface. We unfortunately do not have any independent proof of the existence of an inversion domain there. However, we do note that prior workers have found that it is relatively probable for inversion domains to form immediately beside an open-core dislocation [13]: the open-core dislocation itself forms by the coalescence of misaligned neighboring islands, and if one of these islands has inverted structure then an inversion domain will result. Thus, we can conclude that it is at least not unlikely that an inversion domain will form beside an open-core dislocation. This fact, together with the very good agreement in the observed structures with that expected from the diffraction results obtained from the (0001) surface, leads us to conclude that the structure observed in Fig. 2 is indeed likely to be that 
that of the " $1 \times 1$ " reconstruction whose details are frozen in place due to the small size of the inversion domain which contains it. Further observations of other "frozen in" structures (possibly obtained using low temperature measurements) are required to conclusively identify this novel reconstruction.

\section{Growth kinetics on bare surfaces}

The surface morphology of GaN films during growth is affected by a number of factors. First, the dislocation density in the films can significantly affect the morphology. Step flow growth leads to the formation of spiral growth mounds around screw dislocations (see e.g. Fig. 1 of Ref. [14]). Also, dislocations in the films can lead to the formation of "pits" on the film surface [15], and extended planar defects may lead to larger morphological features on the surface. A typical example is shown in Fig. 3(a), which is an atomic force microscope (AFM) image of a Ga-polar GaN film grown on SiC. The dislocation density in these films in moderately high $\left(10^{9}-10^{10} \mathrm{~cm}^{-2}\right.$ range), and the morphology seen in Fig. 3(a) is typical of that obtained on MBE-grown GaN films. In contrast, for growth by metal-organic chemical vapor deposition (MOCVD), the surface morphologies are flatter, due both to a reduced density of dislocations and possible differences in the kinetic process which gives rise to the morphological variations near the dislocations (e.g. preferential sublimation of material around the dislocations during growth) [15].

RHEED observation during MBE growth of GaN reveals a characteristic behavior as one varies between growth under relatively Ga-rich and N-rich conditions. In the former, the RHEED pattern is streaky, indicative of flat morphology, and in the latter the RHEED pattern is spotty indicative of rough surface morphology. This behavior has been noted by a number of authors [6,16], and indeed, the transition from smooth to rough is generally used as a definition of Ga-rich compared to N-rich growth. AFM imaging has been used to image this smooth to rough behavior in real-space [16]; our results for this morphological change are pictured in Fig. 3. Figure 3(a), as described above, shows the result for Ga-rich growth; the inset shows the associated streaky RHEED pattern. Figure 3(b) gives the result for growth under N-rich conditions. The overall surface morphology (dominated by the effects of dislocations) is similar to that of Fig. 3(a), but on an $30 \mathrm{~nm}$ length-scale a distinct roughness of the surface can be seen and this roughness give rise to the spotty RHEED pattern shown in the inset. The results shown in Fig. 3 are for Ga-polar films, although very similar results have been obtained for the case of N-polar films [17].

An explanation for the smooth to rough behavior has been advanced by Zywietz et al. [18]. They consider diffusion barriers for $\mathrm{Ga}$ and $\mathrm{N}$ atoms on both (0001) and (0001) surfaces, using first-principles theoretical computations. They find firstly that the diffusion barriers are substantially less for $\mathrm{Ga}$ atoms compared to $\mathrm{N}$ atoms. Furthermore, the barrier for the faster moving species $(\mathrm{Ga})$ is substantially increased on a N-terminated surface compared to a Ga-terminated surface. The latter result would lead to the roughening behavior seen in experiment, assuming that the surface is terminated by at least some $\mathrm{N}$ under $\mathrm{N}$-rich conditions. However, this assumption is, at least for the $(000 \overline{1})$ surface, not consistent with either experiment or theory since they both find a N-terminated bilayer to be energetically unstable [3]. Nevertheless, it is suggested by Zywietz et al. that a kinetic accumulation of $\mathrm{N}$ during growth may occur on the surface, since the diffusion rate of $\mathrm{N}$ is sufficiently slow that it may not have time to find another $\mathrm{N}$ atom thereby forming $\mathrm{N}_{2}$ and desorbing from the surface. In this case, the surface concentration of $\mathrm{N}$ would indeed build up during growth, thereby leading to the reduction in the Ga diffusion rate and the corresponding on- 
set of rough growth.

\section{Indium-covered $(000 \overline{1})$ surfaces}

In addition to studies of bare GaN surfaces, we have also examined the surface structures resulting from co-deposition of indium as performed during the growth of InGaN alloys. Measurements have been performed to date only on N-polar films, i.e. with (000 $\overline{1})$ surfaces [19]. Surface morphology of the InGaN films is determined using STM and AFM, the surface indium content is estimated using Auger spectroscopy, and the bulk In concentration is determined using X-ray diffraction (XRD).

STM results for two different InGaN films are shown in Fig. 4. The film pictured in Fig. 4(a) was grown at a relatively high temperature of $670^{\circ} \mathrm{C}$ and with an $\mathrm{In} /(\mathrm{In}+\mathrm{Ga})$ flux ratio of 0.20 . Consequently it had negligible $(<1 \%$, from XRD) In in the bulk. Auger spectroscopy, however, indicated a large amount of In on the surface. As seen in Fig. 4(a), individual atomic species are visible on the surface, with $1 \times 1$ order, although there are two types of atoms visible - one bright and the other dark, with 0.2-0.3 A height difference between them. The bright atoms account for about $70 \%$ of the surface coverage. Prior studies using first-principles theory have demonstrated that In atoms, incorporated into a $1 \times 1$ adlayer on the $(000 \overline{1})$ surface will have surface height about $0.2 \AA$ higher than that of Ga atoms in the adlayer, thus leading to the interpretation of the bright atoms in the STM images being In and the dark atoms being Ga. The large surface concentration of In arises from surface segregation of this species during growth, due to the much smaller In- $\mathrm{N}$ bond strength compared to that of Ga-N [19].

Figure 4(b) shows an STM image obtained from a film grown at $660^{\circ} \mathrm{C}$, with an $\mathrm{In} /(\mathrm{In}+\mathrm{Ga})$ flux ratio of 0.36 . XRD measurements indicate about $5 \%$ indium incorporation in the bulk. Again the surface layer consists primarily of indium atoms. However, in contrast to Fig. 4(a), the image of Fig. 4(b) displays several different types of atomic features on the surface, labeled A-E in the image. Atoms of type A are, we believe, the only Ga atoms on the surface. All the rest of the atoms, except possibly those of type $\mathrm{E}$, are indium atoms. A typical surface In atom, type $\mathrm{B}$, is higher than the Ga atoms by about $0.10-0.15 \AA$; this height difference is less than the $0.2-0.3 \AA$ seen in Fig. 4(a), although the fact that the Ga atoms in Fig. 4(b) are single, isolated atoms will tend to make them appear somewhat higher (brighter) in the STM images. The type C In atoms in Fig. 4(b) appear brighter than those of type B, which we attribute to the presence of subsurface metal atoms. In the metal layer below the surface there are both indium and gallium atoms, and the indium atoms there will push up the surface indium atoms above them and make then look brighter in the STM images. The type D In atoms (surrounding the surface Ga atoms) are higher in the STM topograph by about $0.05 \AA$ than the type B In atoms. This height difference is consistent with theoretical results indicating a $0.04 \AA$ height difference between In atoms in a $2 \times 2$ mixed adlayer $(0.5 \mathrm{ML}$ In + $0.5 \mathrm{ML} \mathrm{Ga}$ ) compared to In atoms in a purely In adlayer [20]. Finally, the type E atoms visible on the surface are adsorbates on top of the surface adlayer, arising from additional metal atoms or possibly from unintentional contaminant atoms.

Regarding the rough to smooth transition observed for the bare surface, the presence of indium during growth seems to have relatively little effect on this transition. In other words, the critical $\mathrm{Ga} / \mathrm{N}$ flux ratio at which this transition occurs seems to be almost independent of the In flux. It is not clear at present why the transition has this dependence. In any case, for growth of InGaN, 
significant incorporation of indium only occurs in a relatively $\mathrm{N}$-rich growth regime. A qualitative reason for this dependence has been previously advanced [19], namely, that weakly adsorbed Ga atoms will tend to displace In atoms in the top layer of the growing InGaN film thereby reducing the In incorporation, but in the N-rich growth regime the concentration of these weakly adsorbed Ga atoms is minimal and thus significant In incorporation can be achieved. Thus, when growing InGaN with significant indium content, the RHEED pattern is generally spotty during growth. Recently, Widmann et al. have proposed that indium has a surfactant effect on the growth of GaN and InGaN [21]. Their result appears to be somewhat opposite from ours, since we find actually a rougher morphology during the InGaN growth. Nevertheless, we do agree with their result that the indium strongly segregates to the surface. We note that the results of Widmann et al. apply probably to Ga-polar films (they used an AlN buffer layer, which tends to produce Ga-polar material [6]), whereas our result is for N-polar films. Future studies of Ga-polar InGaN films are planned, in order to more completely understand the role of indium in the growth kinetics.

\section{Conclusions}

In summary, we find first of all that the surface kinetics of bare GaN surfaces are dominated by the presence or absence of surface Ga layers. Most reconstructions of the surface do contain terminating Ga layers, leading to fast surface diffusion and smooth morphology during growth under Garich conditions. For growth under N-rich conditions, it is believed that $\mathrm{N}$ can accumulate on the surface, giving rise to the observed rough morphology in that case. Indium adsorbates are found to effectively substitute for the surface gallium during growth of $\mathrm{InGaN}$, because of the strong surface segregation of the indium. The transition point between smooth and rough morphology is determined primarily by the $\mathrm{Ga} / \mathrm{N}$ flux ratio even in the presence of $\mathrm{In}$.

\section{Acknowledgments}

We thank J. Neugebauer, J. Northrup, and T. Zywietz for useful discussions. This work was supported by grants from the Office of Naval Research, N00014-96-1-0214, and the National Science Foundation, DMR-9615647.

[1] A. R. Smith, R. M. Feenstra, D. W. Greve, M.-S. Shin, M. Skowronski, J. Neugebauer, and J. Northrup, Appl. Phys. Lett. 72, 2114 (1998).

[2] W. E. Packard, J. D. Dow, R. Nicolaides, K. Doverspike, and R. Kaplan, Superlattices and Microstructures 20, 145 (1996); J. Mater. Res. 12, 646 (1997).

[3] A. R. Smith, R. M. Feenstra, D. W. Greve, J. Neugebauer, J. E. Northrup, Phys. Rev. Lett. 79, 3934 (1997).

[4] A. R. Smith, R. M. Feenstra, D. W. Greve, M. S. Shin, M. Skowronski, J. Neugebauer, and J. Northrup, J. Vac. Sci. Technol. B 16, 2242 (1998).

[5] A. R. Smith, R. M. Feenstra, D. W. Greve, M. S. Shin, M. Skowronski, J. Neugebauer and J. Northrup, Surf. Sci. 423, 70 (1999).

[6] R. Held, D. E. Crawford, A. M. Johnson, A. M. Dabiran, and P. I. Cohen, J. Electron. Mater. 26, 272 (1997); R. A. Held, G. Nowak, B. E. Ishaug, S. M. Seutter, A. Parkhomovsky, A. M. Dabiran, P. I. Cohen, I. Grzegory, and S. Porowski, J. Appl. Phys. 85, 7697 (1999).

[7] Y.-C. Chao, C. B. Stagarescu, J. E. Downes, P. Ryan, K. E. Smith, D. Hanser, M. D. Bremser, and R. F. Davis, Phys. Rev. B 59, R15586 (1999). 
[8] V. M. Bermudez, D. D. Koleske, and A. E. Wickenden, Appl. Surf. Sci. 126, 69 (1998).

[9] A. R. Smith, R. M. Feenstra, D. W. Greve, J. Neugebauer, and J. Northrup, Appl. Phys. A 66, S947 (1998).

[10] V. Ramachandran, C. D. Lee, R. M. Feenstra, A. R. Smith, and D. W. Greve, submitted to Phys. Rev. Lett. (Comment on Q. K. Xue, Q. Z. Xue, R. Z. Bakhtizin, Y. Hasegawa, I. S. T. Tsong, T. Sakurai, and T. Ohno, Phys. Rev. Lett. 82, 3074 (1999)).

[11] V. Ramachandran, C. D. Lee, R. M. Feenstra, A. R. Smith, J. E. Northrup, and D. W. Greve, to appear in J. Cryst. Growth.

[12] V. Ramachandran, M. F. Brady, A. R. Smith, R. M. Feenstra and D. W. Greve, J. Electron. Mater. 27, 308 (1998).

[13] D. Cherns, W. T. Young, J. W. Steeds, F. A. Ponce, and S. Nakamura, J. Cryst. Growth 178, 201 (1997).

[14] A. R. Smith, V. Ramachandran, R. M. Feenstra, D. W. Greve, M. S. Shin, M. Skowronski, J. Neugebauer and J. Northrup, J. Vac. Sci. Technol. A 16, 1641 (1998).

[15] P. J. Hansen, Y. E. Strausser, A. N. Erikson, E. J. Tarsa, P. Kozodoy, E. G. Brazel, J. P. Ibbetson, U. Mishra, V. Narayanamurti, S. P. DenBaars, and J. S. Speck, Appl. Phys. Lett. 72, 2247 (1998).

[16] E. J. Tarsa, B. Heying, X. H. Wu, P. Fini, S. P. DenBaars, and J. S. Speck, J. Appl. Phys. 82, 5472 (1997).

[17] A. R. Smith, V. Ramachandran, R. M. Feenstra, D. W. Greve, A. Ptak, T. H. Myers, W. L. Sarney, L. Salamanca-Riba, M. -S. Shin and M. Skowronski, MRS Internet J. Nitride Semicond. Res. 3, 12(1998).

[18] T. Zywietz, J. Neugebauer, and M. Scheffler, Appl. Phys. Lett. 73, 487 (1998).

[19] H. Chen, A. R. Smith, R. M. Feenstra, D. W. Greve, and J. E. Northrup, MRS Internet J. Nitride Semicond. Res. 4S1, G9.5 (1999).

[20] T. Zywietz, Ph.D. Thesis, Technical University of Berlin, 1999.

[21] F. Widmann, B. Daudin, G. Feuillet, N. Pelekanos, and J. L. Rouvière, Appl. Phys. Lett. 73, 2642 (1998). 
(a)

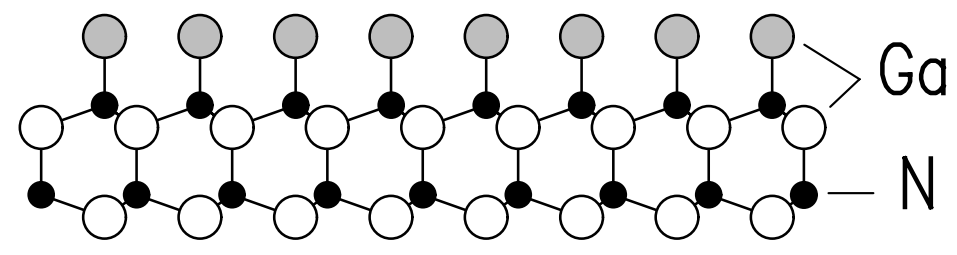

$\uparrow(000 \overline{1})$

(b)

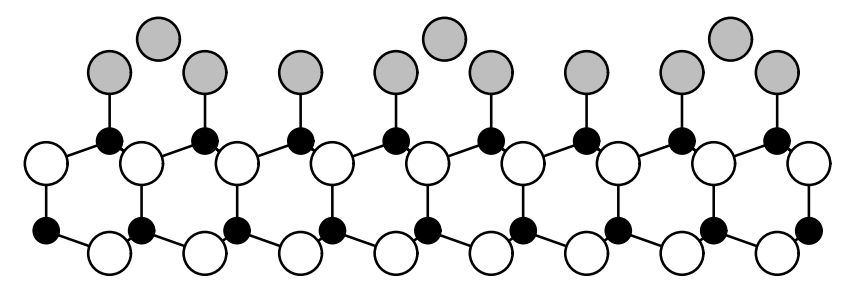

(c)

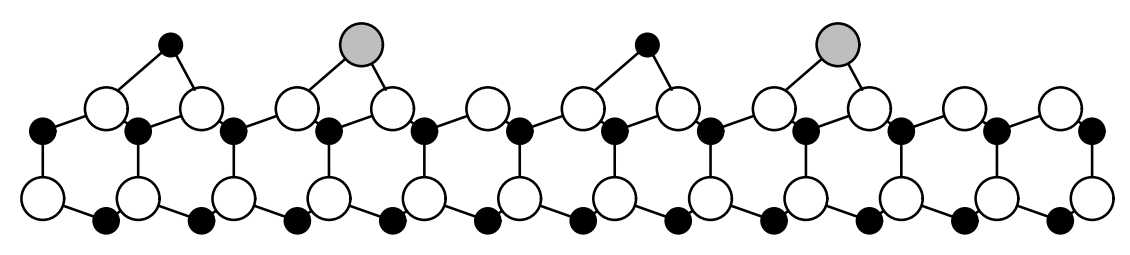

$\uparrow(0001)$

(d)

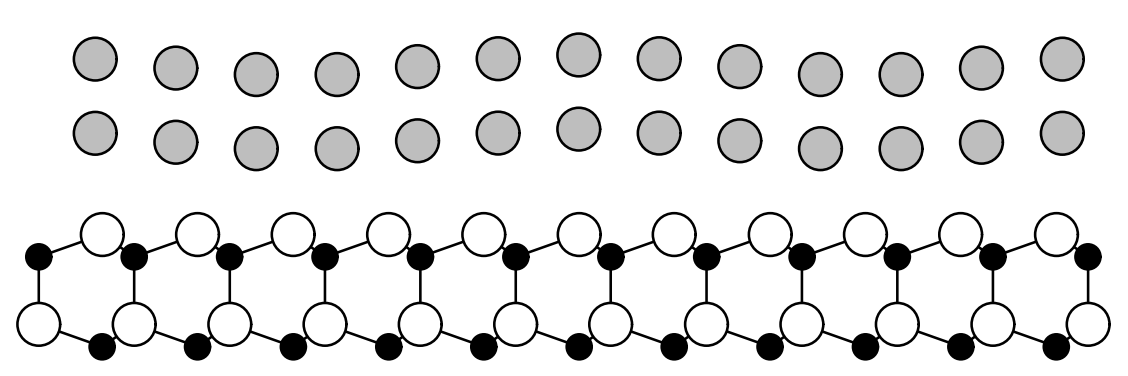

Figure 1 Schematic illustration of surface reconstructions occurring on GaN (0001) and (000 $\overline{1})$ surfaces, in the N-rich and the Ga-rich limits. (a) Ga adlayer forming $1 \times 1$ structure on the $(000 \overline{1})$ surface; (b) additional Ga adatoms forming weakly bound arrangements on this $1 \times 1 \mathrm{Ga}$ adlayer; (c) an arrangement of $\mathrm{Ga}$-adatoms and $\mathrm{N}$-adatoms on the (0001) surface; (d) incommensurate double layer of $\mathrm{Ga}$ adatoms on the (0001) surface (the vertical displacements in the double layer are shown exaggerated, for clarity). 

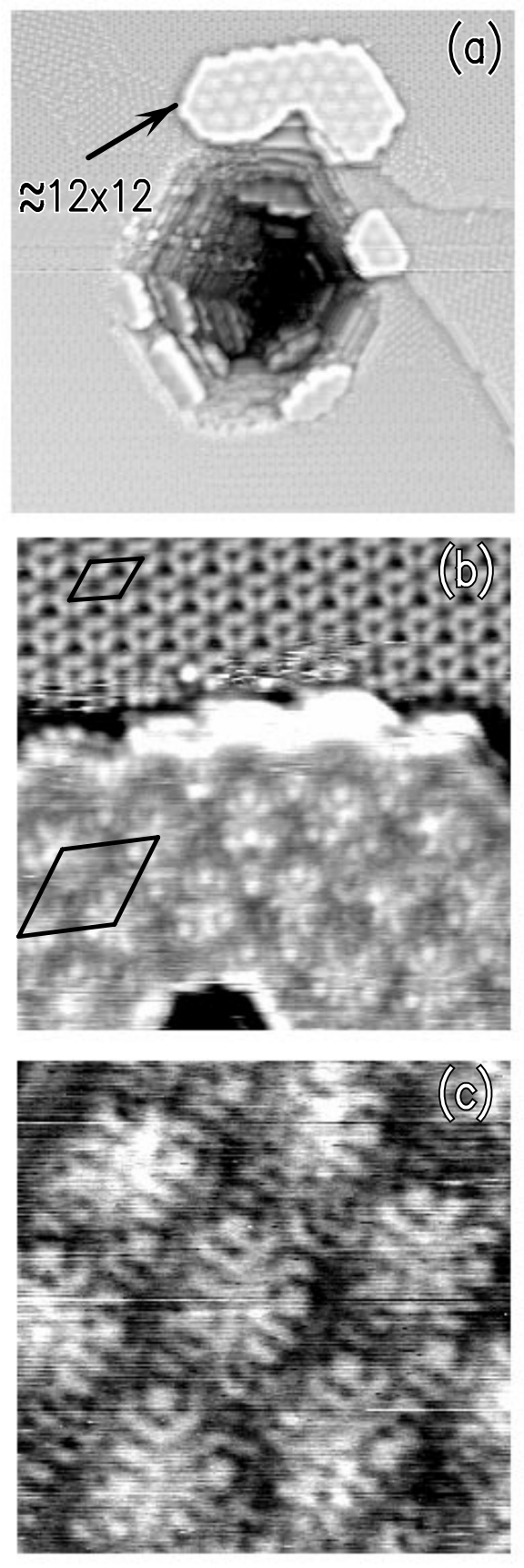

Figure 2 STM images obtained in the vicinity of a surface pit on the (000 $\overline{1})$ surface of GaN. (a) $90 \times 90 \mathrm{~nm}^{2}$ image showing surface pit and surrounding surface region, including an anomalous reconstructed region with symmetry $\approx 12 \times 12$. (b) $22 \times 22 \mathrm{~nm}^{2}$ expanded view of the $\approx 12 \times 12$ region together with $6 \times 6$ region above it, with dark lines showing unit cells of each. (c) $11 \times 11 \mathrm{~nm}^{2}$ further expanded view of the $\approx 12 \times 12$ region. Sample bias voltage for all images is $1.0 \mathrm{~V}$ and tunnel current is $0.09 \mathrm{nA}$. 


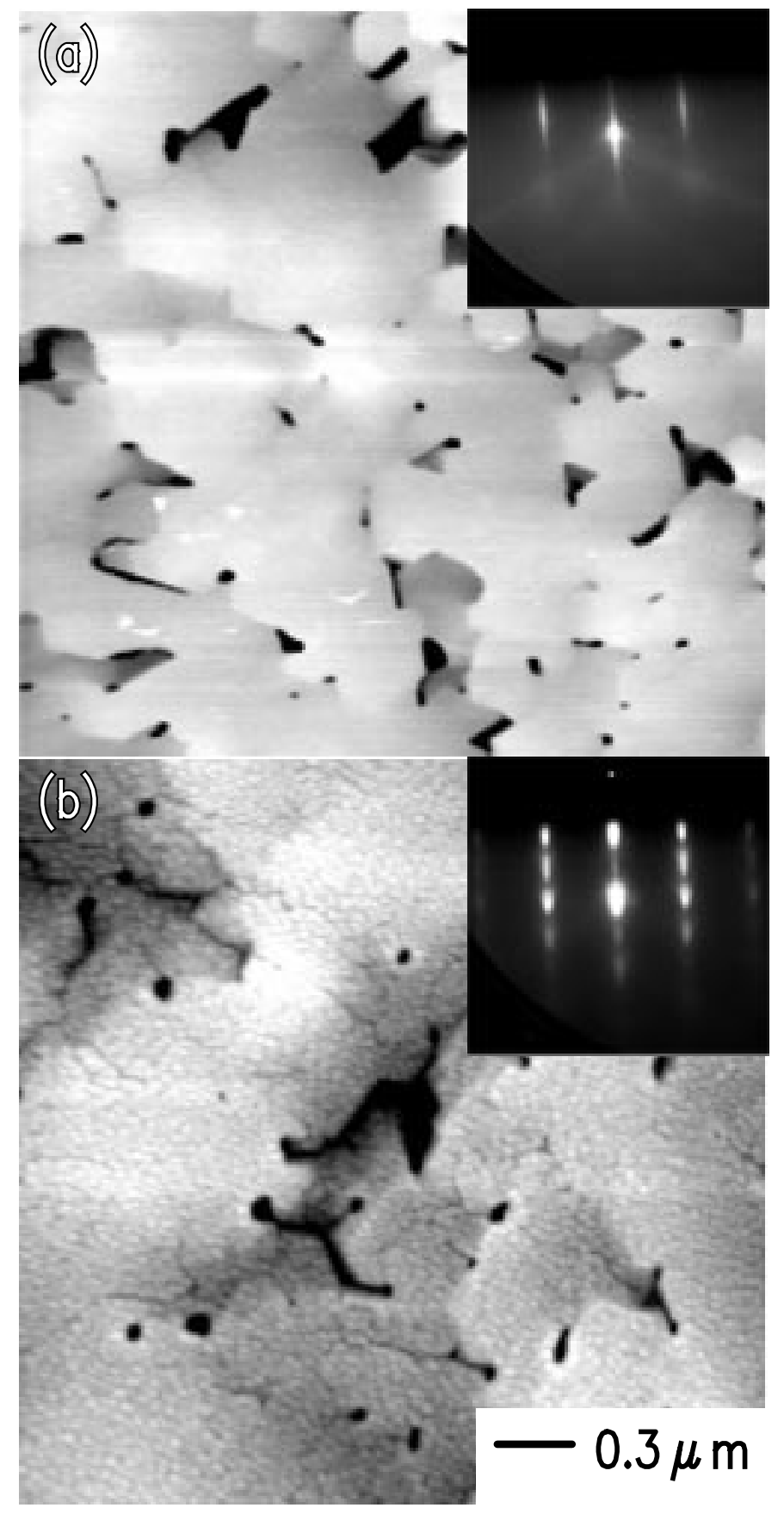

Figure 3 AFM images of Ga-polar GaN films on $\mathrm{SiC}$ : (a) grown under Ga-rich condition, and (b) grown under N-rich conditions. The insets show the RHEED patterns for each film, taken with the electron beam along a [1120] direction. 

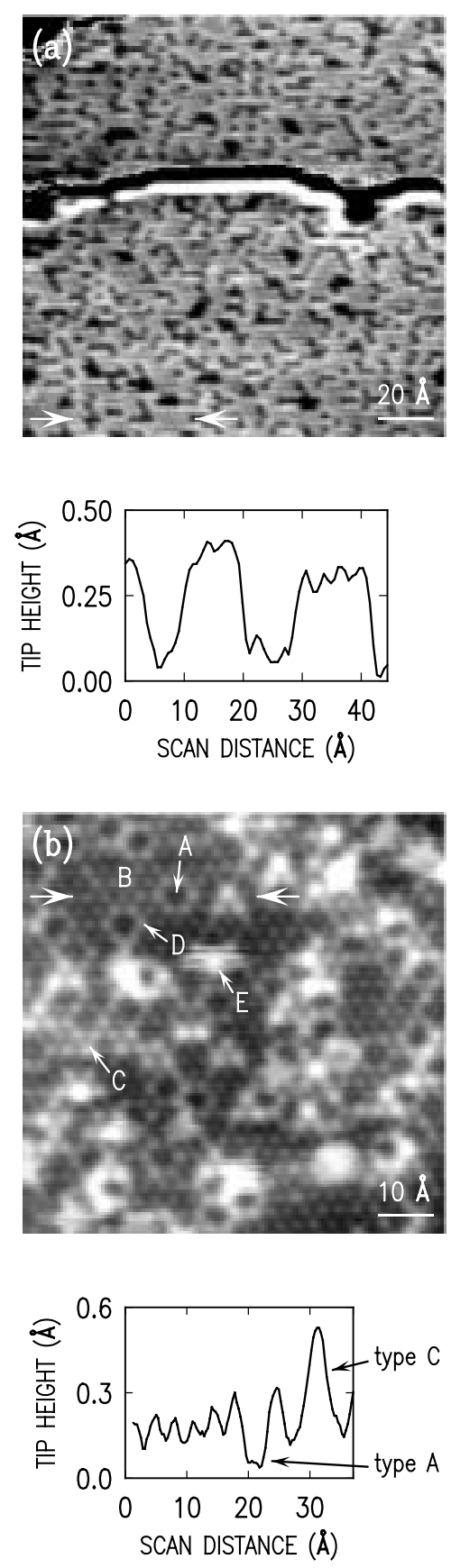

Figure 4 STM images of the $\operatorname{InGaN}(000 \overline{1})$ surface. (a) Film with negligible indium incorporation in the bulk. Image obtained with sample bias voltage of $-0.5 \mathrm{~V}$ and tunnel current of $0.075 \mathrm{nA}$. The image contains two terraces, displayed with a split grey-scale having $0.6 \AA$ range on each terrace; the step separating the terraces extends horizontally through the center of the image. (b) Film with about $5 \%$ indium incorporation in the bulk. Image obtained with sample bias of $-1.0 \mathrm{~V}$ and tunnel current of $0.15 \mathrm{nA}$. Grey-scale range is $0.6 \AA$. Symbols A-E denote different types of atoms, as described in the text. The large arrows indicate the positions of line cuts, shown below each image. 